\title{
Synchronous Push-Pull Class E Rectifiers with Load-Independent Operation for Megahertz Wireless Power Transfer
}

Huang, Xiaosheng; Dou, Yi; Lin, Shuyi; Tian, Yuan; Ouyang, Ziwei; Andersen, Michael A. E.

Published in:

IEEE Transactions on Power Electronics

Link to article, DOI:

10.1109/TPEL.2020.3038814

Publication date:

2021

Document Version

Peer reviewed version

Link back to DTU Orbit

Citation (APA):

Huang, X., Dou, Y., Lin, S., Tian, Y., Ouyang, Z., \& Andersen, M. A. E. (2021). Synchronous Push-Pull Class E Rectifiers with Load-Independent Operation for Megahertz Wireless Power Transfer. IEEE Transactions on Power Electronics, 36(6), 6351 - 6363. https://doi.org/10.1109/TPEL.2020.3038814

\section{General rights}

Copyright and moral rights for the publications made accessible in the public portal are retained by the authors and/or other copyright owners and it is a condition of accessing publications that users recognise and abide by the legal requirements associated with these rights.

- Users may download and print one copy of any publication from the public portal for the purpose of private study or research.

- You may not further distribute the material or use it for any profit-making activity or commercial gain

- You may freely distribute the URL identifying the publication in the public portal 


\title{
Synchronous Push-Pull Class E Rectifiers with Load-Independent Operation for Megahertz Wireless Power Transfer
}

\author{
Xiaosheng Huang, Member, IEEE, Yi Dou, Student Member, IEEE, Shuyi Lin, Yuan Tian, Ziwei Ouyang, Senior \\ Member, IEEE, and Michael A.E. Andersen, Member, IEEE,
}

\begin{abstract}
This paper presents the analytical modeling of synchronous class $\mathbf{E}$ rectifiers with the load-independent operation, which achieves zero-phase-angle input impedance, soft-switching over the entire load range with a constant voltage gain. The optimal design of the synchronous class $E$ rectifier is proposed to realize both of zero-voltage-switching turn-on and zero-currentswitching turn-off at the expected output power. An $L C C-S$ compensated MHz-WPT topology, which comprises the push-pull class $E$ inverter and rectifier with the load-independent operation, is proposed to achieve the fully soft-switching and a nearlyconstant voltage gain over the entire load range. The efficiency improvement of the compensation network is also investigated to provide a design methodology for the proposed topology. A 6.78MHz WPT prototype, along with an alternative phase detector using an auxiliary coil to realize phase detection, is built to validate the analytical model and the proposed methodology. The system efficiency reaches $86.7 \%$ at 214 watts output. The synchronous push-pull class $\mathbf{E}$ rectifier maintains soft-switching over the load range, and the rectification efficiency reaches $94.6 \%$.
\end{abstract}

Index Terms-wireless power transfer, class $\mathrm{E}$, synchronous rectifier, load-independent

\section{NOMENCLATURE}

$\omega$
$i_{a c}$
$I_{a c}$
$v_{a c}$
$V_{a c}$
$\varphi$
$v_{S}$
$v_{S n}$
$i_{S}$
$i_{S n}$
$i_{L f}$
$I_{L \pi}$
$p_{\pi}$

Angular frequency.

Input current of rectifier.

Amplitude of $i_{a c}$.

Input voltage of rectifier.

Amplitude of $v_{a c}$.

Phase shift of $i_{a c}$.

Switch voltage.

$=\frac{v_{S}}{V_{d c}}$, normalized switch voltage.

Switch current.

$=\frac{i_{S}}{I_{d c r}}$, normalized switch current.

Current passing through $L_{f}$.

Current passing through $L_{f}$ at $\omega t=\pi$. $=\frac{I_{L \pi} \cdot \omega L_{f}}{V_{d c}}$, normalzied $I_{L \pi}$.

This work was supported in part by the National Natural Science Foundation of China under Grant 51607039, in part by the Natural Science Foundation of Fujian Province of China under Grants 2019J01772 and 2018J01623, and in part by the Program for the Training of Distinguished Young Scientists in Fujian Provincial Universities under Grant GY-Z18161. (Corresponding author: Ziwei Ouyang.)

Xiaosheng Huang, Shuyi Lin, and Yuan Tian are with the School of Electronic, Electrical Engineering and Physics, Fujian University of Technology, Fuzhou 350118, China (e-mail: hxs@ fjut.edu.cn; linshuyi1985@qq.com; tianyuan8162@qq.com).

Yi Dou, Ziwei Ouyang, and Michael A.E. Andersen are with the DTU Elektro, Technical University of Denmark, 2800 Kongens Lyngby, Denmark (e-mail: yidou@elektro.dtu.dk; zo@elektro.dtu.dk; ma@elektro.dtu.dk). $q$

$p$

$p_{\text {optm }}$

$R_{\text {optm }}$

$R_{r}$

$G_{r e c}$

$I_{d c r}$

$P_{o r}$

$G_{r e c \_d}$

$G_{\text {inv_d }}$

$G_{\text {mag }}$

$P_{O_{-} d}$

$P_{\text {or }} d$

$R_{r_{-} d}$

$k_{23}$

$k_{t r}$

$k_{e q}$

$R_{1 \sim 3}$

$Q_{1 \sim 3}$

$Q_{L}$

$R_{d c o}$ $=\frac{1}{\omega \sqrt{L_{f} C_{f}}}$, normalized resonant frequency of $L_{f}$ and $C_{f}$.

$=\frac{I_{a c} \cdot \omega L_{f}}{V_{d c}}$, load factor of rectifier.

Optimal rated value of $p$.

Load resistance mapping to $p_{\text {optm }}$.

Rated load resistance of rectifier.

Voltage gain of rectifier.

Rated output current of rectifier.

Rated output power of rectifier.

Voltage gain of push-pull rectifier.

Voltage gain of push-pull inverter.

Voltage gain of resonant tank.

Output power of push-pull rectifier.

Rated output power of push-pull rectifier.

Rated load resistance of push-pull rectifier.

Coupling coefficient between $L_{2}$ and $L_{3}$.

Coupling coefficient between $L_{t}$ and $L_{r}$.

$=\sqrt{L_{1} / L_{2}}$, equivalent coupling coefficient between $l o_{0} p_{1}$ and $l_{o o p}$ of resonant tank.

ESR of resonance $l o o p_{1 \sim 3}$. $=\frac{\omega L_{1 \sim 3}}{R_{1 \sim 3}}$, resonant quality factors of $l o o p_{1 \sim 3}$. $=\frac{\omega L_{3}}{R_{a c}}$, loaded quality factor of resonant tank.

Load resistance of receiver.

\section{INTRODUCTION}

Wireless power transfer (WPT) is getting more attractive for the applications of power electronics (e.g., portable devices, medical implants, electric vehicles, etc.) [1]-[5]. For WPT systems based on magnetic coupling, increasing operation frequency usually means smaller magnetic components and higher induced voltage. It is conducive to reduce the system size, extend the transfer distance, and enhance misalignment tolerance. However, the increased frequency brings a challenge to high-efficiency and high-reliable MHz-WPT systems because of the substantial losses of the switches and the resonant tank. Up to now, the issues on high-frequency rectifiers are attracting more attention as the rectification loss constitutes a large portion of the system losses [6]-[13].

Various topologies can realize the high-frequency rectification, such as full-bridge [14], class E [15], class DE [16], class EF [17], class F [18]. Due to the increased switching losses, the rectification efficiency may distinctly reduce when working in the $\mathrm{MHz}$ region. Moreover, the parasitic inductance and capacitance may cause serious ringing (i.e., high-frequency 
harmonics) at the instant of switching. This phenomenon not only leads to additional losses but also resulting in electromagnetic interference issues. Therefore, the soft-switching resonant rectifiers are required to overcome the above issues.

Class $\mathrm{E}$ resonant rectifiers are attractive for $\mathrm{MHz}-\mathrm{WPT}$ applications due to its simplicity and low harmonic contents [19]. The conventional half-wave class E rectifiers can achieve low-voltage-derivation switching (i.e., the diode turns off at zero $d v_{D} / d t$ and turns on at low $d v_{D} / d t$ ) by using a capacitor in parallel with the diode [20], [21]. A similar approach can be implemented to achieve low-current-derivation switching (i.e., the diode turns on at zero $d i_{D} / d t$ and turns off at low $\left.d i_{D} / d t\right)$ by using an inductor in series with the diode [22]. Besides, the harmonic contents can be further reduced by interleaving two half-wave rectifiers, which constitute a full-wave class $\mathrm{E}$ rectifier. Both of the class $\mathrm{E}$ half-wave and full-wave rectifiers for $\mathrm{MHz}$-WPT systems were discussed [7], [9]. However, the inherent forward voltage of diodes will cause high conducting losses, which limit the applications of class $\mathrm{E}$ rectifiers.

The synchronous rectification is a promising way to reduce the losses caused by diode-based rectifiers. Class $\mathrm{E}$ and its derivative topologies (e.g., class EF, push-pull class E), which comprise only low-side driven switches, are preferred for MHz-WPT systems. Different from the diodes which block the reverse current, the synchronous switches are able to conduct bidirectionally. Thus, the synchronous rectifiers can operate as time-reversed inverters. Synchronous class E rectifiers can be found in many DC-DC converters with the operation frequency from $\mathrm{kHz}$ to $\mathrm{GHz}$ [23]-[27]. However, so far, there are few articles discussed the synchronous class $\mathrm{E}$ rectifiers for $\mathrm{MHz}-$ WPT systems, of which the resonant tank is different from that of conventional DC-DC converters [8]-[13].

The phase shift control is usually required to achieve softswitching of synchronous rectifiers by adjusting the switching phase over the load range [10]. Generally, two kinds of methods can directly realize the phase detection, i.e., the voltagecomparator-based [8] and the current-transformer-based [9], [10]. As in [8], a voltage comparator is implemented to detect the threshold of the switch voltage and generate a fixedwidth pulse. However, the waveform of the switch voltage changes as the load varies, and resulting in an unexpected switching phase shift. This issue will be more troublesome in the differential (i.e., push-pull) class $\mathrm{E}$ topology due to the unbalance voltage waveforms [28]. In [9], the zero-current detection is implemented to generate the gate driving signal of several hundred $\mathrm{kHz}$. However, it is challenging for the existing current transformers to work in the $\mathrm{MHz}$ region due to the bandwidth limitation. The self-oscillating resonant gate driver is another potential solution to drive the synchronous switches [24]. However, the switching phase is also sensitive to the load variation since the driver is energized by an alternating voltage node of the rectifier.

The existing studies still lack a preferable method to driving the synchronous switches avoiding the unexpected phase shift caused by the load variation. Consequently, the load-independent class $\mathrm{E}$ rectifiers are preferable to simplify the control since the optimal switching phase is constant. Theoretically, the parameters of the synchronous rectifiers are

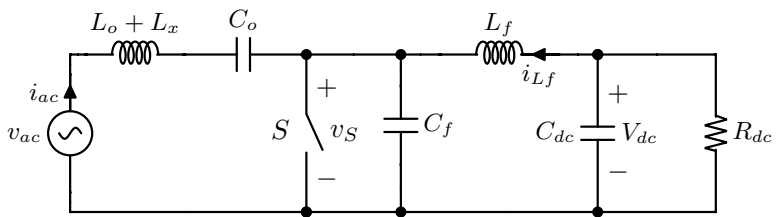

Fig. 1. Synchronous class-E rectifier with finite inductance. The output inductor $L_{f}$, parallel capacitor $C_{f}$ and external inductor $L_{x}$ construct a loadnetwork, which shapes the switch voltage to achieve soft-switching or other features. The input filter $\left(L_{o}\right.$ and $\left.C_{o}\right)$ resonates at the operation frequency.

identical with that of the inverters. However, modeling the load-independent operation from the perspective of inversion can not provide a concise method to obtain the impedance features and the circuit parameters of load-independent class $\mathrm{E}$ rectifiers. The rectification efficiency and the voltage gain are highly dependent on the impedance features, which are related to load variation. Due to the lack of detailed analytical modeling in the existing literature, it is difficult to analyze and optimize the load-independent synchronous class E rectifiers for MHz-WPT applications.

This paper investigates the analytical modeling of synchronous class $\mathrm{E}$ rectifiers with the load-independent operation from the perspective of rectification. It provides a straightforward method to analysis and design the load-independent synchronous class E rectifiers. Based on the model, the implementation for the proposed MHz-WPT topology is also presented. Section II presents the detailed analytical derivation of the single-switch synchronous class E rectifiers with the load-independent operation, which achieves both of zerophase-angle (ZPA) input and soft-switching over the entire load range with a constant voltage gain. The optimal design is proposed according to the analytical expressions deducted. Then, Section III proposes an $L C C-S$ compensated $\mathrm{MHz}-$ WPT topology combined with the load-independent push-pull synchronous class E rectifier, which is derived from the singleswitch one. The improved design method of the compensation network is also investigated to provide a design methodology for the proposed topology. In Section IV, a design example is presented and simulated. Then, in Section V, the hardware implementation is also illustrated regarding the inverter, the rectifier, the compensation network and the phase detector avoiding the unexpected phase shift of conventional methods. The 6.78-MHz WPT prototype is built and tested to validate the analytical model and the optimization method proposed. Finally, the conclusions are given in Section VI.

\section{Analytical Modeling and Design Optimization OF LOAD-INDEPENDENT SYNCHRONOUS CLASS E RECTIFIERS}

The concept of load-independent class $\mathrm{E}$ inverters was introduced in [29]. The modeling of the load-independent synchronous class $\mathrm{E}$ rectifiers can begin with the derivation of switch voltage, which is similar to modeling the inverters.

\section{A. Switch Voltage}

Fig.1 illustrates the topology of the synchronous classE rectifier. It is assumed that the switch has zero on- 
resistance and infinite off-resistance. The switch is ON during $0<\omega t \leq \pi$ and OFF during $\pi<\omega t \leq 2 \pi$. The equivalent series resistance (ESR) of passive components is ignored. The input current, which is filtered by the input filter with high enough $\sqrt{L_{o} / C_{o}}$, can be expressed by

$$
i_{a c}(\omega t)=I_{a c} \cdot \sin (\omega t+\varphi)
$$

The on-state switch current during $0<\omega t \leq \pi$ is given by

$$
i_{S}(\omega t)=I_{L \pi}+\frac{V_{d c}}{\omega L} \cdot(\omega t-\pi)+i_{a c}(\omega t)
$$

When the switch is in the off-state, $C_{f}$ is charged by $i_{a c}$ and $i_{L f}$. Thus, the switch voltage for $\pi<\omega t \leq 2 \pi$ meets

$$
\omega C_{f} \cdot \frac{d v_{S}}{d \omega t}=\frac{1}{\omega L} \int_{\pi}^{\omega t}\left(V_{d c}-v_{S}\right) d \omega t+I_{L \pi}+i_{a c}(\omega t)
$$

Differentiating both sides gives

$$
\frac{1}{q^{2}} \frac{d^{2} v_{S n}}{d \omega t^{2}}-p \cdot \cos (\omega t+\varphi)+v_{S n}-1=0
$$

Thus, the general solution of the normalized switch voltage is

$$
v_{S n}(\omega t)=1+\xi_{1} \cos (q \omega t)+\xi_{2} \sin (q \omega t)-\xi_{3} q \cos (\omega t+\varphi)
$$

where

$$
\xi_{3}=\frac{p q}{1-q^{2}}
$$

The coefficients $\xi_{1}$ and $\xi_{2}$ can be obtained by solving the expressions for ZVS turn-off, i.e.,

$$
\begin{gathered}
v_{S n}(\pi)=0 \\
\left.\frac{d v_{S n}(\omega t)}{d \omega t}\right|_{\omega t=\pi}=q^{2} p_{\pi}+q^{2} p \cdot \sin (\varphi)
\end{gathered}
$$

Then, $\xi_{1}$ and $\xi_{2}$ are given by

$$
\begin{aligned}
\xi_{1}= & -\xi_{3}[\sin (q \pi) \sin (\varphi)+q \cos (q \pi) \cos (\varphi)] \\
& -q \sin (q \pi) \cdot\left(p_{\pi}+p \sin (\varphi)\right)-\cos (q \pi) \\
\xi_{2}= & \xi_{3}[\cos (q \pi) \sin (\varphi)-q \sin (q \pi) \cos (\varphi)] \\
& +q \cos (q \pi) \cdot\left(p_{\pi}+p \sin (\varphi)\right)-\sin (q \pi)
\end{aligned}
$$

Solving the equation for volt-second balance of $L_{f}$ gives

$$
\begin{aligned}
p_{\pi}= & \frac{q \xi_{3} \cos (\varphi)+1}{q \tan \left(\frac{q \pi}{2}\right)^{2}}+\frac{2 q \xi_{3} \sin (\varphi)+\pi}{2 \sin \left(\frac{q \pi}{2}\right)^{2}}-\frac{\xi_{3} \sin (\varphi)}{q} \\
& -p \sin (\varphi)
\end{aligned}
$$

Consequently, the normalized switch voltage is expressed as a function of 3 variables, i.e., $q, p$ and $\varphi$.

\section{B. Criteria of Load-Independent Operation}

Fourier series can be applied to analyze the voltage gain and input impedance. The fundamental harmonic of $v_{S n}$ can be separated into the active $\left(V_{R n}\right)$ part and the reactive $\left(V_{X n}\right)$ part given by

$$
\begin{aligned}
& V_{R n}=\frac{1}{\pi} \int_{\pi}^{2 \pi} v_{S n}(\omega t) \sin (\omega t+\varphi) d \omega t \\
& V_{X n}=\frac{1}{\pi} \int_{\pi}^{2 \pi} v_{S n}(\omega t) \cos (\omega t+\varphi) d \omega t
\end{aligned}
$$

To figure out the switch voltage with load variation, deriving (11) and (12) with respect to $p$ gives

$$
\begin{gathered}
\frac{d V_{R n}}{d p}=\left[\frac{2 q^{4} \cot \left(\frac{q \pi}{2}\right)^{2}}{\pi\left(q^{2}-1\right)^{2}}\right] \cdot \sin (\varphi)^{2} \\
\frac{d V_{X n}}{d p}=\left[\frac{q^{4} \cos (\varphi)(\cos (q \pi)+1)}{\pi\left(q^{2}-1\right)^{2} \sin \left(\frac{q \pi}{2}\right)^{2}}\right] \cdot \sin (\varphi) \\
+\frac{2 q^{3} \cot \left(\frac{q \pi}{2}\right)}{\pi\left(q^{2}-1\right)^{2}}+\frac{q^{2}}{2\left(q^{2}-1\right)}
\end{gathered}
$$

Let $\varphi=\pi$, (13) and (14) can be simplified as

$$
\left.\frac{d V_{R n}}{d p}\right|_{\varphi=\pi}=0
$$

which means $V_{R n}$ is irrelevant to load variation. And,

$$
\xi_{4}=\left.\frac{d V_{X n}}{d p}\right|_{\varphi=\pi}=\frac{2 q^{3} \cot \left(\frac{q \pi}{2}\right)}{\pi\left(q^{2}-1\right)^{2}}+\frac{q^{2}}{2\left(q^{2}-1\right)}
$$

As $q$ is constant for physical rectifiers, $V_{X n}$ is proportional to the input current. The voltage gain of the rectifier meets

$$
\frac{1}{G_{r e c}}=\frac{V_{a c}}{V_{d c}}=\left(\xi_{4}+\frac{L_{x}}{L_{f}}\right) \cdot p+V_{R_{n}}
$$

Thus, $V_{X n}$ can be compensated by fixed inductance given by

$$
L_{x}=-\xi_{4} L_{f}
$$

Therefore, by applying the particular values of $\varphi$ and $L_{x}$, the compensated input impedance is purely resistive. A constant load-independent voltage gain is achieved and given by

$$
G_{r e c}=\frac{1}{\frac{2}{\pi}+\frac{q}{\left(1-q^{2}\right) \tan \left(\frac{\pi q}{2}\right)}}
$$

To achieve ZVS turn-on at $\omega t=2 \pi$, the $q$ can be obtained by numerically solving

$$
v_{S n}(2 \pi)=2+\frac{\sin (q \pi)\left(q \pi+2 q^{2} \xi_{3} \sin (\varphi)\right.}{2 \sin \left(\frac{q \pi}{2}\right)^{2}}=0
$$

where $\varphi=\pi$. Thus,

$$
q=1.291547
$$

Then, (16) gives

$$
\xi_{4}=-0.266228
$$

The load-independent voltage gain is given by

$$
G_{\text {rec }}=0.629125
$$

Consequently, (21) and (22) give the normalized parameters which are identical for the synchronous class $\mathrm{E}$ rectifiers achieving the load-independent operation. It realizes ZVS turnon and ZPA input impedance over the entire load range with the constant voltage gain. 


\section{Design Optimization}

In order to design a practical rectifier, the rated parameters have to be calculated to satisfy the required efficiency and the expected output specifications. In this subsection, an optimal design is proposed to achieve ZVS turn-on and zero-currentswitching (ZCS) turn-off at the rated condition, which achieves the maximum efficiency at the expected output power.

The value of $p$ achieving ZCS turn-off can be obtained by solving

$$
\left.\frac{d v_{S n}}{d \omega t}\right|_{\omega t=\pi}=\frac{\pi q^{2}}{2 \sin \left(\frac{q \pi}{2}\right)^{2}}+q\left(\xi_{3}+1\right) \cot \left(\frac{q \pi}{2}\right)=0
$$

which gives the optimal value of $p$ as

$$
p_{\text {optm }}=1.648457
$$

According to definition of $p$, the $p_{\text {optm }}$ can be rewritten as

$$
p_{\text {optm }}=2 G_{r e c} \cdot \frac{\omega L_{f}}{R_{\text {optm }}}
$$

where $R_{\text {optm }}$ is the optimal load resistance mapping to $p_{\text {optm }}$. The switch voltage under load variation can be obtained by using different values of $p$ mapping to $R_{d c}$. As in Fig.2, the switch maintains ZVS regardless of load variation. When $R_{d c}<R_{o p t m}$, the switch will conduct reversely during a specific interval. Normally, the practical switches (MOSFET, GaN FET, etc.) conduct reversely with a limited voltage. Thus, this phenomenon will cause additional switching losses and also affect the voltage gain. Therefore, in this paper, we use $R_{\text {optm }}$ as the optimal rated $R_{d c}$, i.e., $R_{r}=R_{\text {optm }}$. The synchronous switches can prevent the reverse conducting at off-state over the load range from the rated value to infinite.

Consequently, the optimal parameters of a physical rectifier are obtained by

$$
\begin{gathered}
R_{r}=\frac{V_{d c}^{2}}{P_{o r}} \\
L_{f}=\frac{R_{r} \cdot p_{o p t m}}{2 \omega G_{r e c}} \\
C_{f}=\frac{1}{L_{f} \cdot q^{2} \omega^{2}}
\end{gathered}
$$

Besides, $L_{X}$ is given by (18). The above equations can realize the load-independent class $\mathrm{E}$ rectifiers, which achieve ZPA input impedance and ZVS turn-on over the entire load range with the constant voltage gain. Moreover, the efficiency is expected to reach the maximum value at the rated condition.

Fig.3 illustrates the $i_{S n}$ versus $R_{d c}$, where the normalized switch current is expressed as

$$
\begin{aligned}
& i_{S n}(\omega t)=\frac{i_{S}(\omega t)}{I_{d c r}}= \\
& \begin{cases}\frac{2 G_{r e c}}{p_{\text {optm }}} \cdot p \cdot \sin (\omega t+\varphi)+i_{L f n}(\omega t) & 0<\omega t \leq \pi \\
0 & \pi<\omega t \leq 2 \pi\end{cases}
\end{aligned}
$$

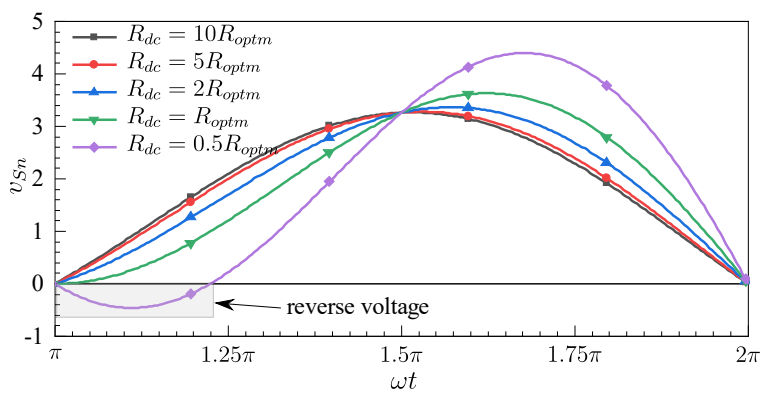

Fig. 2. The normalized switch voltage $v_{S n}$ versus load resistance $R_{d c}$. ZVS turn-on is achieved regardless of load variation. The peak voltage increases as $R_{d c}$ decreases. The peak $v_{S n}$ reaches 4.397 at $R_{d c}=0.5 R_{o p t m}$, compared with 3.631 at $R_{d c}=R_{o p t m}$. The reverse voltage usually causes unexpected conducting of physical switches and affect the load-independent operation.

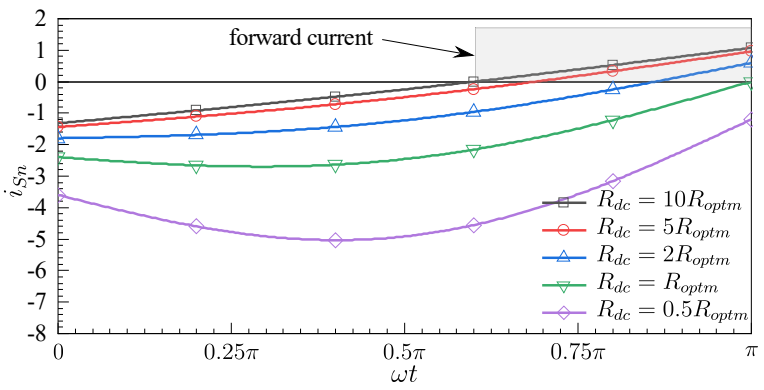

Fig. 3. The normalized switch current $i_{S n}$ versus load resistance $R_{d c}$. The zero-cross instant moves as load varies. The current amplitude increases as $R_{d c}$ decreases (i.e., the output power increases).

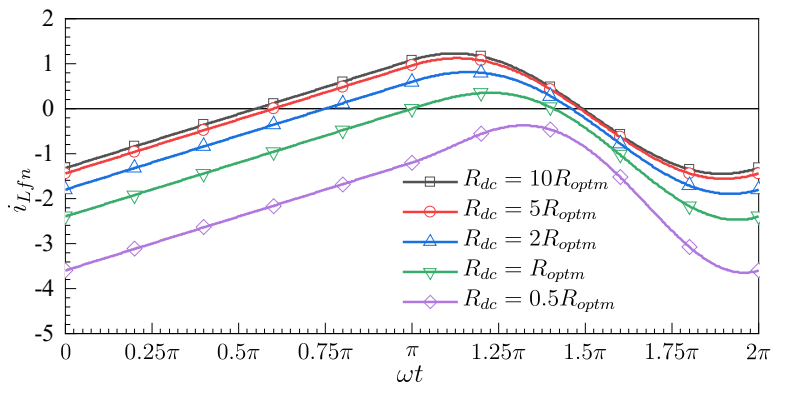

Fig. 4. The normalized inductor current $i_{L f n}$ versus load resistance $R_{d c}$. The peak-peak value of the current is nearly constant as load varies.

where the normalized $i_{L f}$ is given by

$$
\begin{aligned}
& i_{L f n}(\omega t)=\frac{i_{L f}(\omega t)}{I_{D C r}}= \\
& \frac{2 G_{r e c}}{p_{\text {optm }}} \cdot \begin{cases}-\pi+\omega t+p_{\pi} & 0<\omega t \leq \pi \\
p_{\pi}+\int_{\pi}^{\omega t}\left(1-v_{S n}\right) d \omega t & \pi<\omega t \leq 2 \pi\end{cases}
\end{aligned}
$$

As in Fig.3, the direction of the switch current turns into forward during a specific interval. The zero-cross point moves as $R_{d c}$ varies. It means the zero-crossing-detection of the switch current is not applicable for synchronous switching.

As in Fig.4, the current amplitude through $L_{f}$ keeps large over the entire load range. Consequently, the loss of $L_{f}$ takes a significant part in the total loss of the class E rectifier. That 


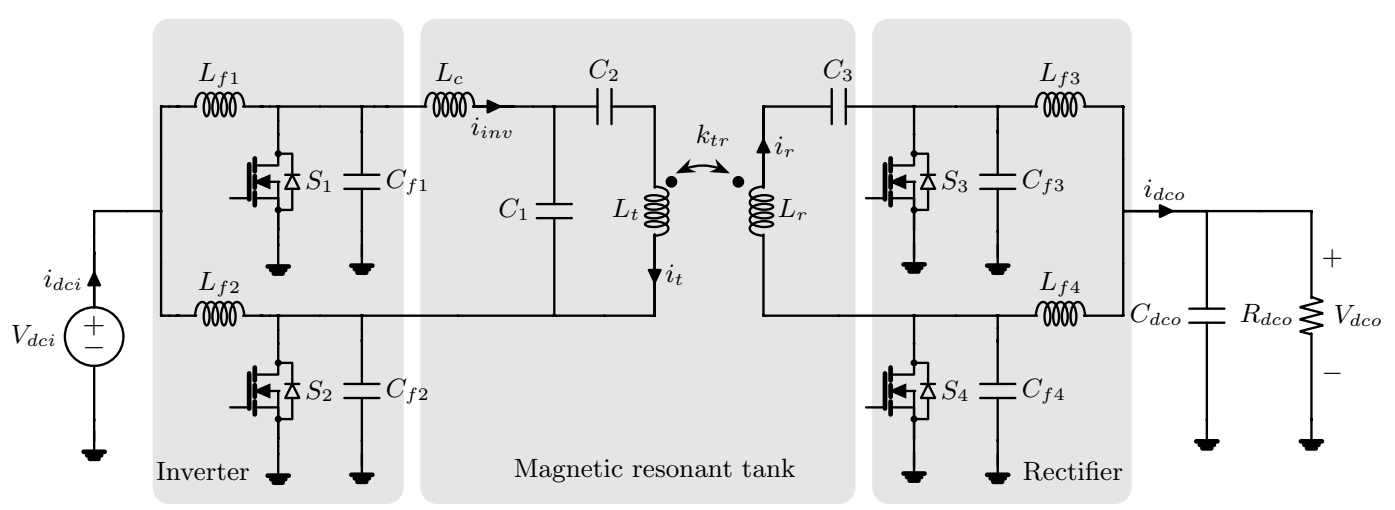

Fig. 5. Proposed WPT topology using the push-pull class E inverter and rectifier with the load-independent operation. Note that $L_{c}=L_{1}+2 L_{x}, L_{t}=L_{2}$ and $L_{r}=L_{3}+2 L_{x}$. The rectifier parameters (i.e., $L_{f}, L_{x}$ and $C_{f}$ ) are also applied to the push-pull class $\mathrm{E}$ inverter to achieve the load-independent operation.

implies the efficiency will be reduced by using a lower rated value of $p$ (i.e., lower $L_{f}$ ).

As a result, the optimal circuit parameters of loadindependent class $\mathrm{E}$ rectifiers, which achieves both of ZVS turn-on and ZCS turn-off at the rated load, can be obtained by (18), (27), (28) and (29).

\section{WPT SYSTEMS USING LOAD-INDEPENDENT Synchronous Push-Pull Class E Rectifier}

As analyzed in Section II, The load-independent class E rectifier can realize soft-switching and ZPA input over the entire load range with the constant voltage gain. The features are preferable for the typical MHz-WPT systems to operate at resonant mode and maintain high-efficiency transfer over the load range. However, the single-switch class E rectifier maintains an $i_{L f}$ of large harmonics over the entire load range, and will result in distinctly voltage ripples and electromagnetic interference. As an alternative solution, the push-pull class $\mathrm{E}$ topology can be implemented to reduce the voltage ripple and suppress harmonic contents.

This section proposes a MHz-WPT topology, of which the inverter and the rectifier are derived from the load-independent operation analyzed in Section II. As illustrated in Fig.5, the push-pull class E topology is implemented for both of the inverter and the rectifier to reduce the harmonic contents and increase the power capability. The inverter and the rectifier are connected by the magnetic resonant tank (i.e., the $L C C-S$ compensated coupling coils). Naturally, the voltage gain of the proposed topology can be calculated by

$$
G_{w p t}=G_{i n v_{-} d} \cdot G_{m a g} \cdot G_{r e c \_d}
$$

where $G_{i n v_{-} d}, G_{m a g}$ and $G_{r e c_{-} d}$ are the voltage gain of the push-pull inverter, the resonant tank, and the push-pull rectifier of the WPT system respectively. The topology of the push-pull class $\mathrm{E}$ inverter is symmetric with that of the rectifier, resulting in $G_{i n v_{-} d}=2 G_{r e c}$ and $G_{r e c_{-} d}=G_{r e c} / 2$. Thus, (32) can be simplified as $G_{w p t}=G_{m a g}$.

It is worth noting that full-bridge rectifiers are not practicable in the proposed WPT topology. The non-resistive input impedance of the full-bridge rectifier will cause detuning operation of the class $\mathrm{E}$ inverter and significantly change the

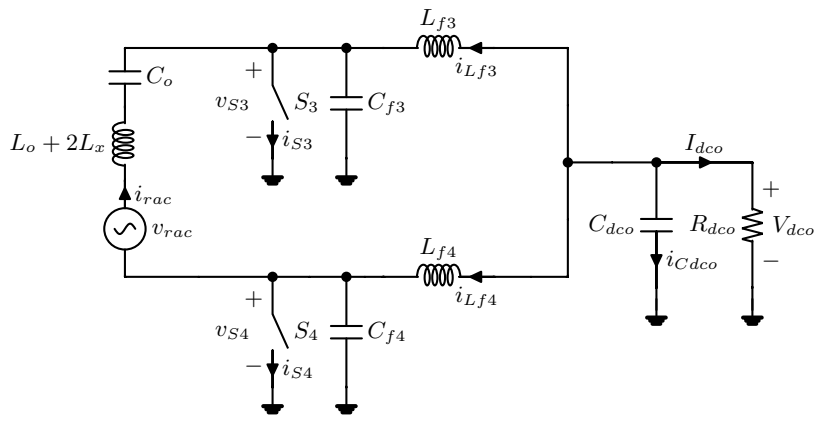

(a) Equivalent circuit

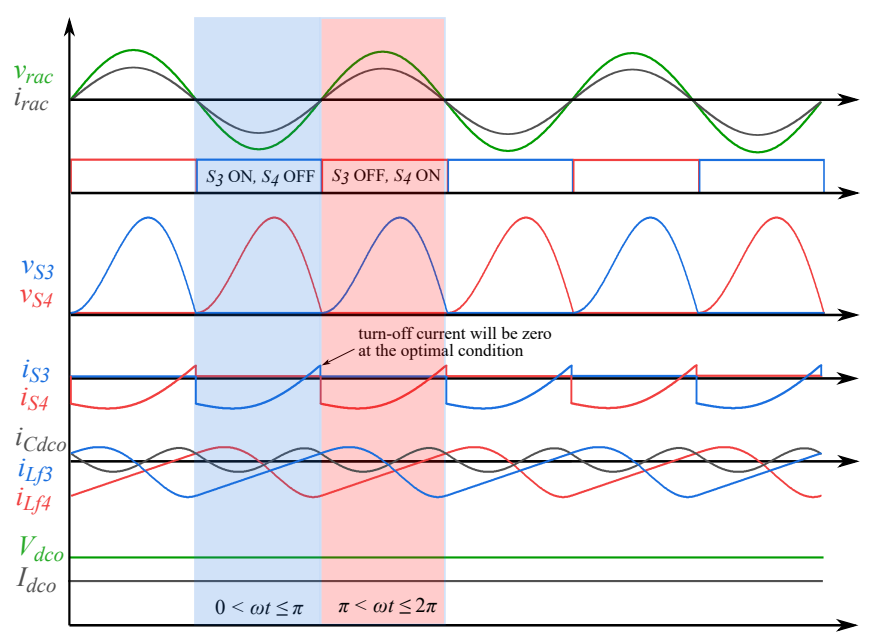

(b) Key waveforms

Fig. 6. Synchronous push-pull class E rectifier with the load-independent operation. The receiving coil combines the input inductance and the external inductance, i.e., $L_{r}=L_{o}+2 L_{x}$. The voltage source $v_{r a c}$ represents the induced EMF (electromotive force) of the receiving coil by the mutual inductance of coupling coils. $S_{3}$ and $S_{4}$ operate differentially with $50 \%$ duty cycle. Due to the interleaving, the current ripple through $C_{d c o}$ is significantly reduced compared with that of the single-switch synchronous class $E$ rectifier.

voltage gain. Moreover, it is also difficult to build a $6.78-\mathrm{MHz}$ full-bridge rectifier which can deliver hundreds of watts due to the parasitic ringing and high harmonic contents.

The proposed WPT topology is well simplified since the first resonant loop acts as an output filter of the inverter, and the third loop acts as an input filter of the rectifier. The 
external inductance $L_{x}$ can be integrated with $L_{1}$ and $L_{3}$ of the compensation network. The analysis and design methodology of the proposed topology are presented in this section as following.

\section{A. Synchronous Push-Pull Class E Topology with Load- Independent Operation}

The push-pull class E rectifier, which comprises two switches operating differentially (i.e., interleaving with 180degree-shift switching), can naturally eliminate the odd-order harmonics at the output node. Thus, it is conducive to suppress the current ripples through the DC capacitor and reduce the electromagnetic interference [9], [28]. Combined with the load-independent operation, the synchronous push-pull class E rectifier can also achieve ZPA input impedance and softswitching over the entire load range. The feature of ZPA impedance is promising to realize a high-efficiency transfer as the magnetic resonant tank maintains resonance at the constant frequency over the entire load range.

As in Fig.6, the rectifier input connects to the seriescompensated resonant loop. The receiving coil provides the input and external inductance of the rectifier, i.e., $L_{o}$ and $L_{x}$. The switch $S_{3}$ is ON during $0<\omega t \leq \pi$ and OFF during $\pi<\omega t \leq 2 \pi$. In reverse, $S_{4}$ is OFF during $0<\omega t \leq \pi$ and ON during $\pi<\omega t \leq 2 \pi$. Since the switches operate differentially, the switching behavior is the same as in a single-switch synchronous class E rectifier. Therefore, the aforementioned optimal design methodology is also applicable to realize the load-independent operation for push-pull class E rectifiers. The parameters can be mapped to single-switch class $\mathrm{E}$ rectifier as in Fig.1, i.e.,

$$
\begin{gathered}
R_{r_{-} d}=\frac{V_{d c}^{2}}{P_{r_{-} d}} \\
L_{f 1,2}=\frac{R_{r_{-} d} \cdot p_{\text {optm }}}{\omega G_{r e c}}=2.620237 \cdot \frac{R_{r_{-} d}}{\omega} \\
C_{f 1,2}=\frac{G_{r e c}}{R_{r_{-} d} \cdot p_{\text {optm }} q^{2} \omega}=0.228791 \cdot \frac{1}{R_{r_{-} d} \cdot \omega}
\end{gathered}
$$

In the proposed WPT topology, the same parameters of the synchronous rectifier (i.e., $L_{f}, L_{x}$ and $C_{f}$ ) are also applied to the inverter. As the synchronous rectifier can be regarded as the time-reversed inverter, the push-pull class E inverter can also achieve the load-independent operation, which achieves softswitching over the entire load range with a constant voltage gain.

\section{B. Magnetic Resonant Tank of WPT Systems}

1) Topology: The resonant tank is formed by the coupling coils and the compensation network. As in Fig.7, the transmitting coil is compensated by an $L C C$ net. The receiving coil is compensated by a capacitor $C_{3}$ in series. Thus, the resonant tank comprises three $L C$ loops resonating at the operation frequency. The parameters meet

$$
\frac{1}{\omega^{2}}=L_{1} C_{1}=L_{2} C_{2 e q}=L_{3} C_{3}
$$

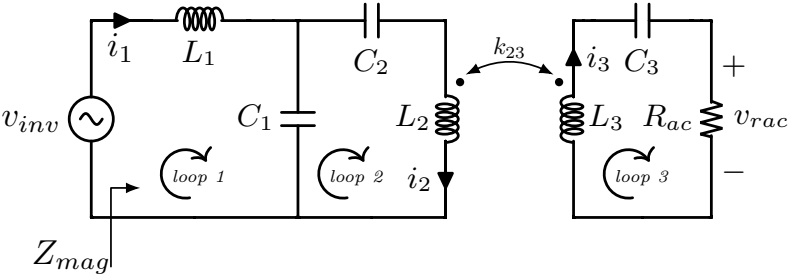

Fig. 7. Magnetic resonant tank formed by the coupling coils and the $L C C-S$ compensation network. $v_{i n v}$ is the output voltage of the inverter. $R_{a c}$ is the input resistance of the rectifier. Note that $L_{x}$ is excluded from the analysis of the resonant tank.

where

$$
C_{2 e q}=\frac{C_{1} C_{2}}{C_{1}+C_{2}}
$$

As the $L_{1}$ and $C_{1}$ resonate at the operation frequency, the current through the transmitting coil can be obtained by Norton's theorem, i.e.,

$$
\dot{I}_{2}=\frac{\dot{V}_{i n v}}{j \omega L_{1}}
$$

which is irrelevant to load resistance. Therefore, the voltage gain is obtained by

$$
G_{\text {mag }}=\frac{\dot{V}_{\text {rac }}}{\dot{V}_{\text {inv }}}=j \omega k_{23} \sqrt{L_{2} L_{3}} \cdot \dot{I}_{2}=\frac{k_{23} \sqrt{L_{2} L_{3}}}{L_{1}}
$$

Thus, the compensation network realizes a load-independent voltage gain. The input impedance of resonant tank can be calculated by

$$
\begin{aligned}
& Z_{\text {mag }}=R_{1}+\frac{k_{e q}^{2} \omega^{2} L_{1} L_{2}}{Z_{l o p 2}} \\
& Z_{l o p 2}=R_{2}+\frac{k_{23}^{2} \omega^{2} L_{2} L_{3}}{R_{3}+R_{a c}}
\end{aligned}
$$

Therefore, the input impedance of the resonant tank is ZPA regardless of load variation, which is preferable for the pushpull class $\mathrm{E}$ inverter.

2) Efficiency Improvement: As the resonant tank is separated into three compensated loops. The efficiency of each loop can be calculated individually, i.e.,

$$
\begin{gathered}
\eta_{1}=\frac{Q_{3}}{Q_{3}+Q_{L}} \\
\eta_{2}=\frac{k_{23}^{2}}{k_{23}^{2}+\frac{1}{Q_{2}}\left(\frac{1}{Q_{3}}+\frac{1}{Q_{L}}\right)} \\
\eta_{3}=\frac{k_{e q}^{2}\left(\frac{1}{Q_{3}}+\frac{1}{Q_{L}}\right)}{k_{e q}^{2}\left(\frac{1}{Q_{3}}+\frac{1}{Q_{L}}\right)+\frac{k_{23}^{2}}{Q_{1}}+\frac{Q_{3}+Q_{L}}{Q_{1} Q_{2} Q_{3} Q_{L}}}
\end{gathered}
$$

Thus, the efficiency of the resonant tank is given by

$$
\eta_{\text {mag }}=\eta_{1} \eta_{2} \eta_{3}
$$

To obtain the proper $L_{1 \sim 3}$, the resonant tank design can begin with selecting $Q_{L}$. Solving zero derivative of (45), i.e.,

$$
\frac{d \eta_{\text {mag }}}{d Q_{L}}=0
$$


gives

$$
Q_{\text {Loptm }}=\frac{\sqrt{Q_{3}} \sqrt{k_{e q}^{2}+\frac{1}{Q_{1} Q_{2}}}}{\sqrt{Q_{2} Q_{3} k_{23}^{2}+1} \sqrt{\frac{k_{e q}^{2}}{Q_{3}}+\frac{k_{23}^{2}}{Q_{1}}+\frac{1}{Q_{1} Q_{2} Q_{3}}}}
$$

where $k_{e q}$ can be re-expressed according to (39), i.e.,

$$
k_{e q}=\sqrt{\frac{k_{23}}{G_{m a g}} \cdot \sqrt{\frac{L_{3}}{L_{2}}}}
$$

where $G_{m a g}$ is given by specifications. $k_{23}$ and $\sqrt{L_{3} / L_{2}}$ are limited by the size and transfer distance of coupling coils. Thus, $L_{3}$ and $R_{a c}$ can be calculated according to the output specifications and $Q_{\text {Loptm }}$. The load resistance of the resonant tank (i.e., the input resistance of the push-pull class E rectifier) is approximately given by

$$
R_{a c}=\frac{2 R_{d c o}}{G_{r e c}^{2}}=5.053074 \cdot R_{d c o}
$$

The rated $R_{a c}$ can be calculated by substituting $R_{d c o}$ with $R_{r_{-} d}$, which is given by application specifications.

To figure out how much efficiency variation the load quality selection can cause, take a parameter set for example, where $Q_{1 \sim 3}=300, k_{23}=0.273$, and $k_{12}=0.412$. The efficiency of the resonant is $95.3 \%$ at $Q_{L}=1$, and reaches $97.1 \%$ at the optimal $Q_{L}=Q_{\text {Loptm }}=3.05$. In other words, the resonant tank loss can be reduced by $39.4 \%$ after load quality optimization in this example. Thus, optimizing $Q_{L}$ is critical for efficiency improvement of the resonant tank.

As in Fig.8, a improved design flowchart is proposed for the resonant tank to adjust the parameters according to measurement. The FEM simulation is used for optimizing the quality factors of the coils by adjusting the cross-sectional diameter and pitch of the wires. Then, the simulated results are used as the initial parameters for each adjustment loop. Finally, the improved design procedure makes the measured practical $Q_{L}$ close to the optimal value as possible, and achieves the optimal efficiency of the resonant tank.

Since the $L_{x}$ of the push-pull class E rectifier/inverter is attached to the physical compensation network, the measured inductance values of resonant loops are $L_{m e s 1}=L_{1}+2 L_{x}$, $L_{m e s 2}=L_{t}=L_{2}$ and $L_{m e s 3}=L_{r}=L_{3}+2 L_{x}$ respectively. Thus, the expected tuned frequency of $l o o p_{1}$ and $l o o p_{3}$ should be adjusted to

$$
f_{m e s 1,3}=\sqrt{\frac{L_{m e s 1,3}-2 L_{x}}{L_{m e s 1,3}}} \cdot f_{o p}
$$

where $f_{o p}$ is the operation frequency. $L_{m e s}$ is the measured inductance. $f_{m e s}$ is the actual tuned frequency of resonant loops. Similarly, the coupling coefficient for resonant tank design is calculated by

$$
k_{23}=k_{t r} \cdot \sqrt{\frac{L_{r}}{L_{r}-2 L_{x}}}
$$

Thus, the coupling coefficient of the practical coupling coils $\left(k_{t r}\right)$ is different from that for resonant tank design $\left(k_{23}\right)$.

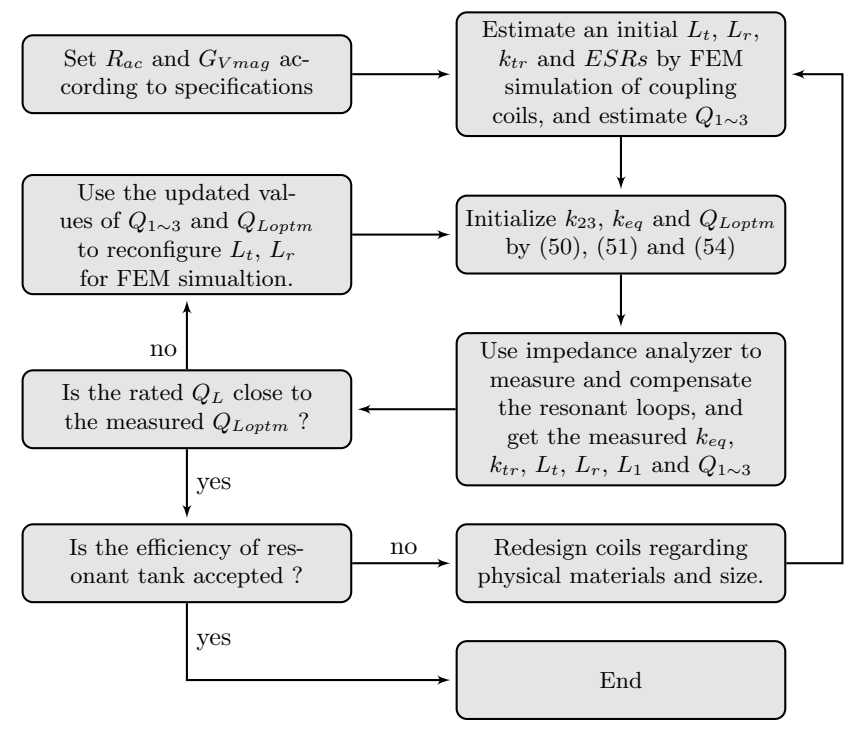

Fig. 8. Efficiency improvement of the $L C C$-S compensated resonant tank. Note that the tuned frequency of $l_{0 o p}$ and $l_{0 o p}$ should be adjusted according to (50). The values of $L_{2}$ and $L_{3}$ are obtained by $L_{t}=L_{2}$ and $L_{r}=L_{3}+2 L_{x}$.

TABLE I

Parameters of Push-Pull Class E Inverter and Rectifier

\begin{tabular}{c|c|c}
\hline \hline & Circuit parameter & Value \\
\hline \multirow{4}{*}{ Inverter } & $V_{d c i}$ & $48 \mathrm{~V}$ \\
& $L_{f 1,2}$ & $644 \mathrm{nH}$ \\
& $L_{x}$ & $171 \mathrm{nH}$ \\
& $C_{f 1,2}$ & $513 \mathrm{pF}$ \\
\hline \multirow{5}{*}{ Rectifier } & $R_{r_{d}}$ & $10.473 \Omega$ \\
& $L_{f 3,4}$ & $644 \mathrm{nH}$ \\
& $L_{x}$ & $171 \mathrm{nH}$ \\
& $C_{f 3,4}$ & $513 \mathrm{pF}$ \\
\hline \multirow{5}{*}{} & Operation frequency & $6.78 \mathrm{MHz}$ \\
& Theoretical rated power & $220 \mathrm{~W}$ \\
& GaN FET $\left(S_{1 \sim 4}\right)$ & TP65H070LDG \\
\hline \hline
\end{tabular}

\section{EXAMPLE Design, HARdWARE IMPLEMENTATION AND SIMULATION}

\section{A. Design and Hardware Implementation}

A prototype working at $6.78 \mathrm{MHz}$ is built to achieve 48 to 48 volt wireless power transfer for unmanned aerial vehicles. Table.I illustrates the circuit parameters, which are obtained as following.

1) Load-Independent Push-Pull Class E Rectifier and Inverter: As in Table.I, the parameters of the inverter and the rectifier are obtained by (33), (34), (35). The theoretical rated power used for calculation is 220 watts for both of the inverter and the rectifier. As mentioned above, since the inverter can be regarded as a time-reversed synchronous rectifier, they use the same parameters to achieve load-independent operation.

Because of the conversation losses, the optimal condition, which achieves ZVS turn-on and ZCS turn-off, is expected to be realized at the output power of about 200 watts which is lower than the theoretical rated output power.

2) Magnetic Resonant Tank: The resonant tank design can begin with the physical size of the coupling coils (i.e., $L_{t}$ and $\left.L_{r}\right)$. In this example, both of the transmitting coil $(15 \mathrm{~cm}$ by 
TABLE II

Parameters of Magnetic Resonant Tank

\begin{tabular}{l|c|cc}
\hline \hline & Parameter & Calc. & Meas. $^{a}$ \\
\hline \multirow{2}{*}{ Transmitting coil $^{b}$} & Outer diameter & \multicolumn{2}{|c}{$150 \mathrm{~mm}$} \\
& Number of turns & \multicolumn{2}{|c}{8} \\
& Pitch & \multicolumn{2}{|c}{$4.5 \mathrm{~mm}$} \\
& Inductance $L_{t}$ & $9.81 \mu \mathrm{H}$ & $10.46 \mu \mathrm{H}$ \\
\hline \multirow{3}{*}{ Receiving coil } & \\
& Outer diameter & \multicolumn{2}{|c}{$100 \mathrm{~mm}$} \\
& Number of turns & \multicolumn{2}{|c}{7} \\
& Pitch & \multicolumn{2}{|c}{$4.2 \mathrm{~mm}$} \\
& Inductance $L_{r}$ & $4.13 \mu \mathrm{H}$ & $4.15 \mu \mathrm{H}$ \\
\hline \multirow{3}{*}{ Compensation } & $L_{1}+2 L_{x}$ & $2.01 \mu \mathrm{H}$ & $2.05 \mu \mathrm{H}$ \\
network & $C_{1}$ & $323.57 \mathrm{pF}$ & $322.70 \mathrm{pF}$ \\
& $C_{2}$ & $62.93 \mathrm{pF}$ & $63.32 \mathrm{pF}$ \\
& $C_{3}$ & $144.93 \mathrm{pF}$ & $145.18 \mathrm{pF}$ \\
\hline & Transfer distance & \multicolumn{2}{|c}{$3 \mathrm{~cm}$} \\
& Operation frequency & \multicolumn{2}{|c}{$6.78 \mathrm{MHz}$} \\
\hline \hline
\end{tabular}

${ }^{a}$ Measured by the impedance analyzer (4294A).

${ }^{b}$ The coupling coils are made of the solid copper wires of $2 \mathrm{~mm}$ diameter.

$15 \mathrm{~cm})$ and receiving coil $(10 \mathrm{~cm}$ by $10 \mathrm{~cm})$ are circular spiral coils fixed in the square covers, and the transfer distance is fixed at $3 \mathrm{~cm}$. Hence, the coupling coefficient is about 0.27 according to an 2D FEM (finite element method) simulation using cylindrical coordinate. In order to realize a conversation ratio of $1: 1$, the $L_{1}$, according to (39), is given by

$$
L_{1}=k_{23} \sqrt{L_{2} L_{3}}
$$

The ratio of $L_{2}$ to $L_{3}$ is about 0.386 . The estimated $Q_{1 \sim 3}$ is 300 . Thus, the optimal loaded quality factor of receiver loop can be calculated as $Q_{\text {Loptm }}=3.047$.

As in Table.II, the parameters of resonant tank can be obtained based on the design flowchart. The measured $k_{e q}, k_{23}$ are $0.403,0.33$ respectively. The measured $Q_{1 \sim 3}$ are 278,216 and 290 respectively. Hence, the actual optimal loaded quality factor is about 2.688 corresponding to the load resistance $R_{d c o}=11.93 \Omega$, which is close to the rated load resistance in Table.I.

3) Phase Detector: In a practical synchronous rectifier, the switches have to be driven with a proper phase shift from the input source. Thus, it requires a phase detector to synchronize the switching phase.

As analyzed in Section II, the load-independent operation achieves the ZPA input of the rectifier over the entire load range with a constant phase shift $\varphi=\pi$. Hence, the synchronous switches can be driven by a pulse with a constant phase shift from the input voltage. Naturally, the phase detection can be achieved without using current transformers or sensing the switch voltage which may cause the unexpected switching phase shift due to load variation.

In this paper, we use an alternative method to realize the phase detection. As illustrated in Fig.9, the auxiliary coil is attached to the receiving coil and indirectly detects the phase of the induced voltage. It is decoupled with the receiving coil but maintains a proper mutual inductance with the transmitting coil. Thus, the induced voltage $\dot{V}_{\text {aux }}$ can be expressed as

$$
\dot{V}_{a u x}=j \omega M_{t a} \dot{I}_{t}+j \omega M_{r a} \dot{I}_{r}
$$

Since $M_{r a}=0$, the detected phase is irrelevant to the receiving coil current, and avoiding the unexpected phase shift.

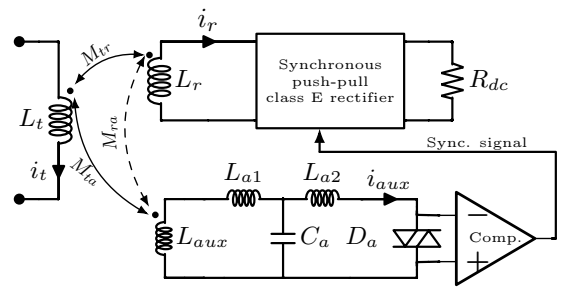

(a)

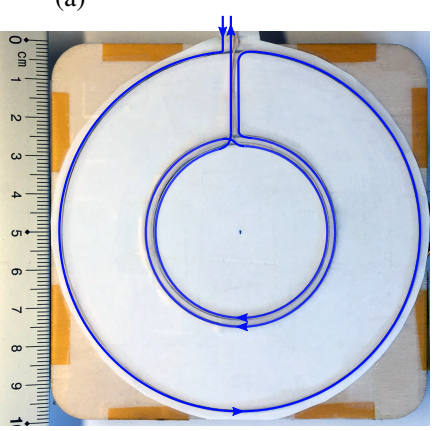

(c)

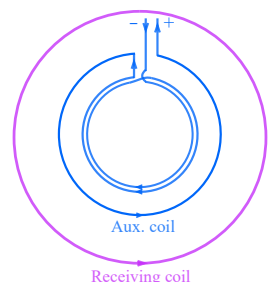

(b)
Fig. 9. Principle of the phase detector using the auxiliary coil. (a) Circuit schematic (b) Coil structure of the receiver (c) Hardware implementation of auxiliary coil. The transmitting coil $L_{t}$ and the receiving coil $L_{r}$ construct the magnetic link of the WPT system. The auxiliary coil comprises 3 turns, of which the winding direction of the outer turn is opposite to the inner 2 turns. The auxiliary coil is adjusted to be decoupled with the receiving coil (i.e., $M_{r a}=0$ ), but maintaining a enough mutual inductance with the transmitting coil (i.e., $M_{t a} \neq 0$ ).

TABLE III

PARAMETERS OF AUXILIARY COIL

\begin{tabular}{c|c}
\hline \hline Parameter & Value \\
\hline Number of turns & 3 \\
Outer diameter of turns & $48 \mathrm{~mm}, 50 \mathrm{~mm}, 100 \mathrm{~mm}$ \\
$M_{t a}$ & $127 \mathrm{nH}$ \\
$L_{a u x}$ & $710 \mathrm{nH}$ \\
Self-resonant frequency & $46.5 \mathrm{MHz}$ \\
\hline \hline
\end{tabular}

The induced voltage of the auxiliary coil has a constant phase shift related to the driving phase of synchronous switches.

As in Table.III, the wire diameter of the auxiliary coil is small enough to neglect the parasitic capacitance and ensure the available operation frequency is much higher than the switching frequency.

As in Fig.9a, the auxiliary coil is compensated by a T-type $L L C$ network, of which parameters meet

$$
\frac{1}{\omega^{2}}=\left(L_{a u x}+L_{a 1}\right) \cdot C_{a}=L_{a 2} \cdot C_{a}
$$

As the auxiliary coil is decoupled with the receiving coil, the current source can be expressed as

$$
\dot{I}_{a u x}=-\omega^{2} M_{t a} C_{a} \cdot \dot{I}_{t}
$$

Thus, the amplitude and phase are irrelevant to the variation of $R_{d c o}$. We use a pair of anti-parallel diodes to limit and shape the output voltage of the T-type network to a nearsquare waveform, of which the rise/fall time is shorter. Then, the comparator generates a synchronization signal sent to the driving circuit of the rectifier. Finally, the switch drivers can be triggered at the expected instant. 
Using series-capacitor is potential compensation for the auxiliary coil. However, the auxiliary will act as a voltage source, which can not drive diodes due to the current limitation. Consequently, the movement of the receiver will result in a wide-range input voltage of the comparator when the diodes are removed. The trigger phase of the comparator will vary as the receiver moves. Moreover, the input voltage may exceed the withstand voltage and degrade the reliability of the signal processing circuit. Thus, it will be complicated for the seriescapacitor compensation to obtain a reliable phase detector.

\section{B. Circuit simulation}

The design example is simulated by MATLAB Simulink with the calculated parameters in the tables above. The synchronization circuit is also included in the schematic to verify the phase detector.

As in Fig.10, a $50 \mu$ s delay is added before the driving pulses of synchronous switches are launched. Thus, the initial output voltage is built by the diode-based rectification. The delay is also implemented in the experimental prototype by using an $R C$ delayer, and provides the following benefits. 1) The $v_{d s}$ overshoot can be well suppressed since the diodebased rectification achieves the soft-switching naturally. 2) The auxiliary power supply for the driving circuit can be powered up adequately regardless of the load conditions, such as deeply discharged batteries. 3) The synchronous rectifier can be activated with a steady phase signal and thus avoiding the interference caused by power-on transient.

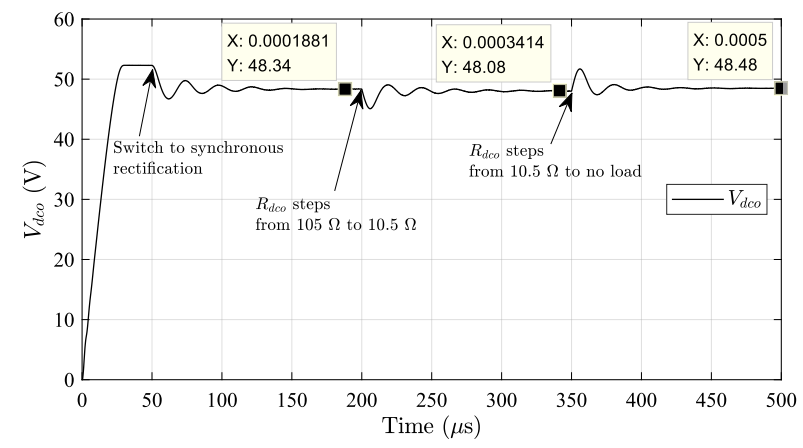

(a) Output voltage

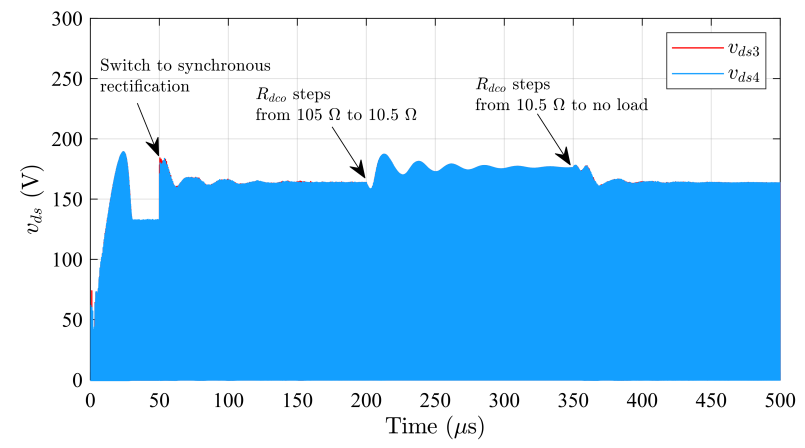

(b) Drain-source voltages

Fig. 10. Simulated output voltage and drain-source voltages of the synchronous push-pull class E rectifier with the load-independent operation. The output voltage varies lower than $0.8 \%$ over entire the load range without using close-loop control.
The transient performance and voltage overshot are related to the $C_{d c o}$, which is a $4.7 \mu \mathrm{F}$ capacitor used in the simulation. As in Fig.10a, a 10-times load step is placed at $200 \mu$ s. The output voltage slightly varies from $48.3 \mathrm{~V}$ to $48 \mathrm{~V}$ as the load resistance steps from $105 \Omega$ to $10.5 \Omega$. In other words, the output voltage varies lower than $0.6 \%$ as the output power steps from $10 \%$ to the rated value. Similarly, the variation is below $0.8 \%$ when the load steps from $10.5 \Omega$ to no load. Therefore, the output voltage is nearly constant regardless of load variation. It also implies that the ZPA input impedance of the rectifier is realized since the system efficiency, the voltage gain, and the soft-switching of the inverter will be affected if the load of the resonant tank is not resistive. The simulation results match well with the theoretical analysis.

Fig.10b illustrates the drain-source voltage of the rectifier, i.e. $v_{d s 3 \sim 4}$. The peak voltage slightly decreases as $R_{d c o}$ reduces. The waveforms present an envelope (i.e., outline) related to the transient state of $V_{d c o}$, and they are unbalanced until the system reaches a steady-state. This phenomenon will be even worse in practical systems due to parameter tolerances. Thus, the conventional phase-detection methods for single-switch rectifiers can not be implemented directly for the synchronous push-pull class E rectifiers. By using the alternative method above, the phase signal is excited by the transmitting coil current. Thus, the driving pulses of synchronous switches are irreverent to the receiving coil current and the load resistance. Fig.11 illustrates $v_{d s}$ with a smaller time-scale. The switches used in the simulation are ideal with linear parasitic capacitance, the $v_{d s}$ shape variation matches well with the theoretical curves in Fig.2.

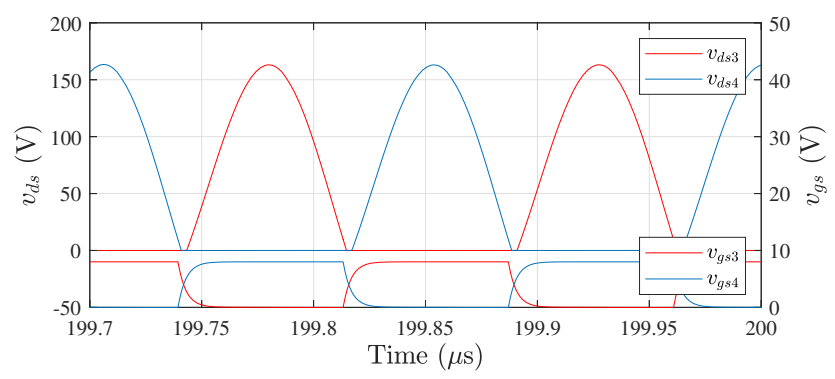

(a) $R_{d c o}=105 \Omega, P_{o_{-} d}=22.3 \mathrm{~W}$

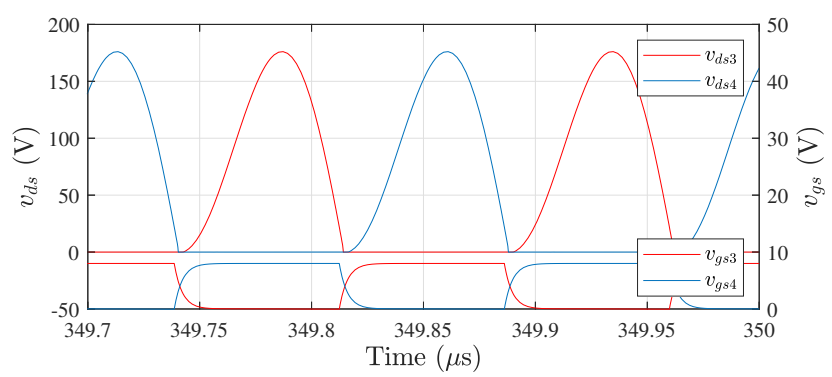

(b) $R_{d c o}=10.5 \Omega, P_{o_{-} d}=219.9 \mathrm{~W}$

Fig. 11. Simulated drain-source voltages. The switches keep ZVS turn-on as load varies. The ZCS turn-off is achieved at the rated load resistance. 


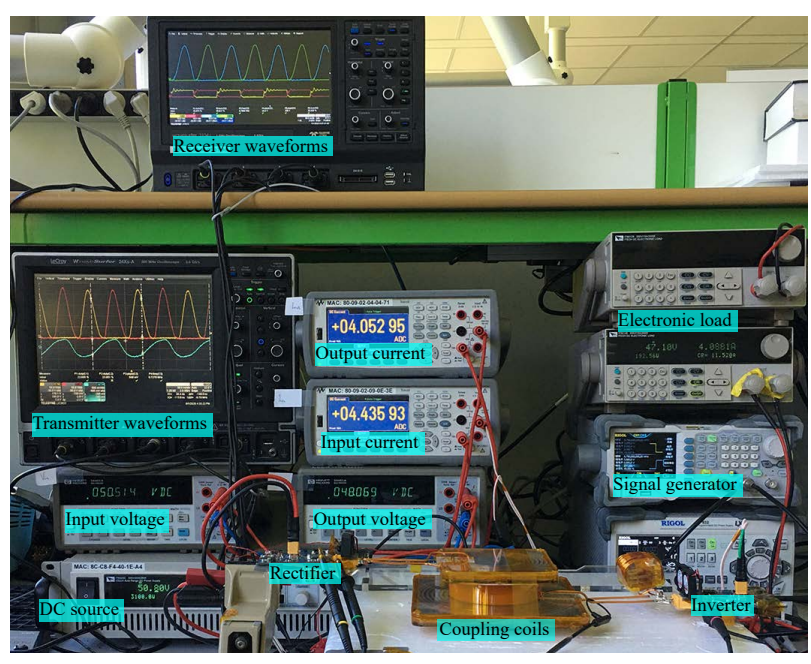

(a) Measurement platform

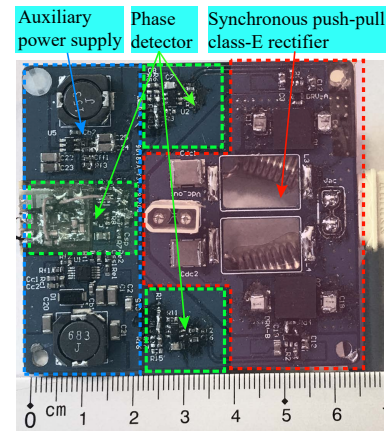

(b) PCB of the active rectifier

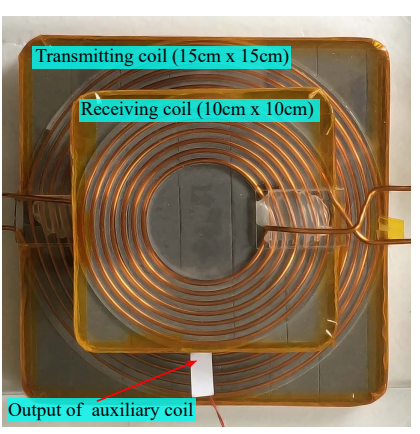

(c) Coil structure
Fig. 12. Experiment setup. The transfer distance is fixed at $3 \mathrm{~cm}\left(k_{t r}=\right.$ 0.316). The auxiliary coil is integrated on the receiving coil.

\section{Experiment Setup AND MEAsurement}

\section{A. Experiment Setup}

Fig.12 illustrates the experiment setup, which is built to verify the design methodology. As in Fig.12b, the circuit board of the rectifier comprises the auxiliary power supply, the phase detector, and the rectification stage. The $L_{f 1 \sim 4}$ are constructed by helical air-core coils, of which ESRs are nearly constant over the load range. When the inverter is powered up, the rectifier operates at diode-based rectification mode until the output voltage of the auxiliary power supply is at steadystate. An $R C$ circuit (on the bottom side of the PCB) is implemented to set the delay before the synchronous driving pulses are enabled. The PCB layout is also implemented for the inverter, excepting its driving pulses are generated by the signal generator. As in Fig.12c, the coupling coils are made of solid copper wires without using magnetic cores. Thus, the quality factors keep constant regardless of the exciting current. The auxiliary coil is decoupled with the receiving coil by adjusting the wire arrangement of the auxiliary coil. It connects to the phase detection circuit to drive the synchronous switches with a proper phase shift as illustrated in Fig.13. Although the prototype is designed for fixed-position transfer, it is verified that the proposed phase detection is also applicable for the synchronous rectification of MHz-WPT systems with variable

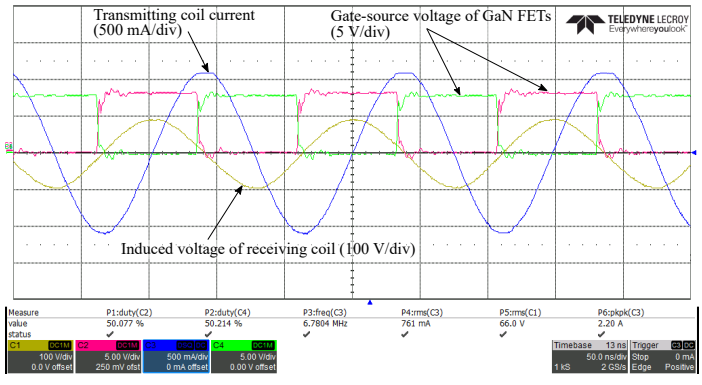

(a) aligned without position shift

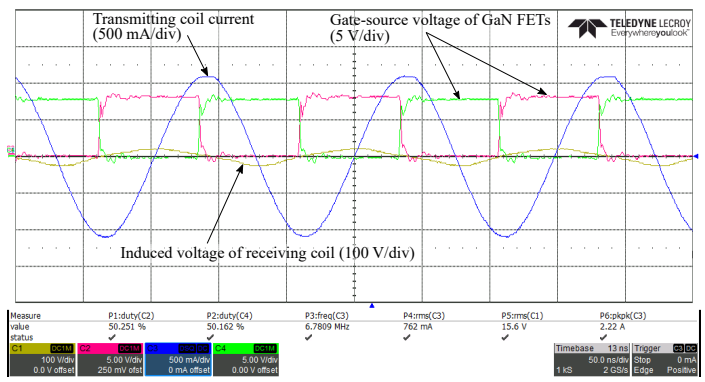

(b) misaligned with a 7-cm position shift

Fig. 13. Driving pulses of the synchronous switches, the transmitting coil current and the open-load voltage of the receiving coil. The phase shift is adjusted according to the propagation delay of the driving circuit.

coupling. The switching phase keeps constant as the receiving coil moves horizontally with respect to the transmitting coil.

\section{B. Measurement}

Fig.14 illustrates the measured $V_{d s}$ and $V_{g s}$ of the synchronous switches. The rectifier maintains ZVS over the designed load range. It matches well with the calculation and simulation in Fig.2 and Fig.11.

As in Fig.14a, the ZVS turn-on is achieved, and the turnoff current is non-zero when the rectifier is at light-load. The turn-off current decrease as the output power increases. As illustrated in Fig.14b, both of the ZVS turn-on and ZCS turnoff are achieved when the output power is close to 200 watts, which is the expected value achieving maximum efficiency. Besides, as illustrated in Fig.14c, the switches begin to conduct reversely at turn-off instant as the output power is above the rated value. Meanwhile, the switches are turning-on before the drain-source voltage decreases to zero. Thus, the unreleased switch voltage will energize the resonance loop formed by the parasitic inductance and the parallel capacitance, resulting in a parasitic ringing phenomenon.

As in Fig.15, the drain-source voltage of the inverter shows a similar variation to that of the rectifier. That means the load impedance of the inverter is resistive or near-resistive.

Fig.16 illustrates the measured efficiency and the voltage gain of the prototype. The efficiency of the resonant tank and the synchronous rectifier are estimated according to the measured quality factors and system efficiency. The measured peak system efficiency reaches $86.7 \%$ at 213.5 watts $\left(R_{d c o}=10.79\right.$ $\Omega$ ), of which the rectifier efficiency also reaches it peak value $94.6 \%$, which is the same as at 194.1 watts. The measured system efficiency is almost the same over the output power 


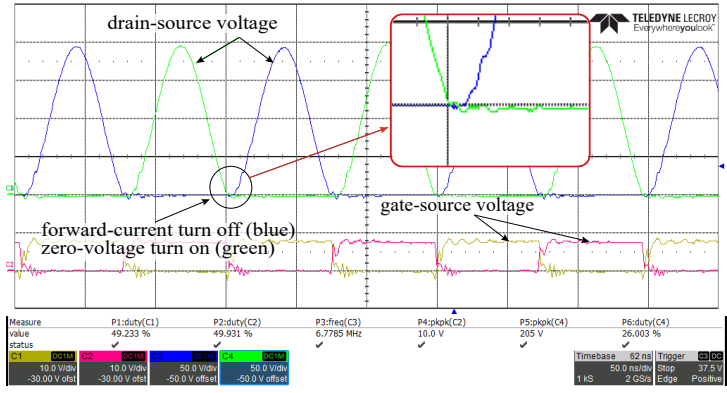

(a) $R_{d c o}=124.73 \Omega, P_{o_{-} d}=18.5 \mathrm{~W}, V_{d c o}=48.02 \mathrm{~V}$

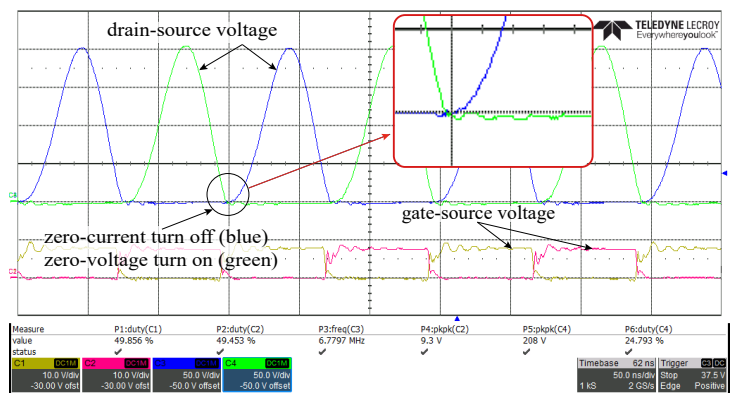

(b) $R_{d c o}=11.84 \Omega, P_{o_{-} d}=194.1 \mathrm{~W}, V_{d c o}=47.95 \mathrm{~V}$

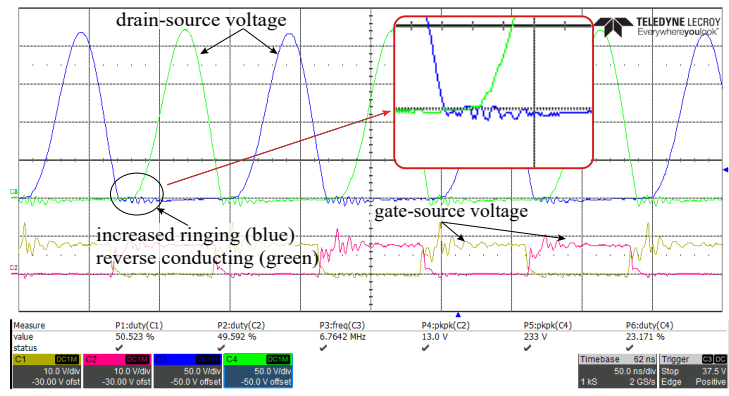

(c) $R_{d c o}=7.88 \Omega, P_{o_{-} d}=291.5 \mathrm{~W}, V_{d c o}=47.94 \mathrm{~V}$

Fig. 14. Measured $V_{d s}$ and $V_{g s}$ of the synchronous switches versus load variation. The soft-switching is maintained over the designed load range, and it trends to be lost when the output power exceeds the rated value.

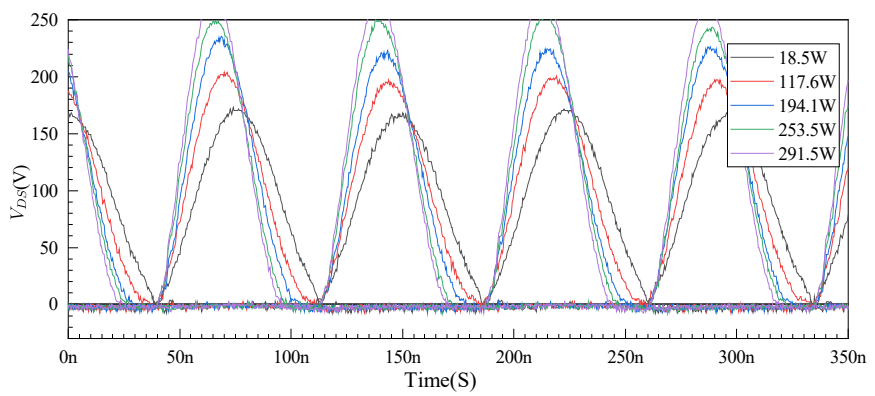

Fig. 15. Measured $V_{d s}$ of the inverter versus load variation. The PCB layout of the rectifier is also implemented for the inverter, of which gate drivers are excited by the signal generator. The drain-source voltage varies as load vaires, similar to the synchronous rectifier.

range from 194.1 to 253.3 watts, and reaches its peak value at about 200 watts, which matches well with the theoretical analysis. When the output power is above the rated value, the efficiency will not step down immediately. However, the temperature of the switches and the inductors will increase sharply as the output power increase to above 300 watts.

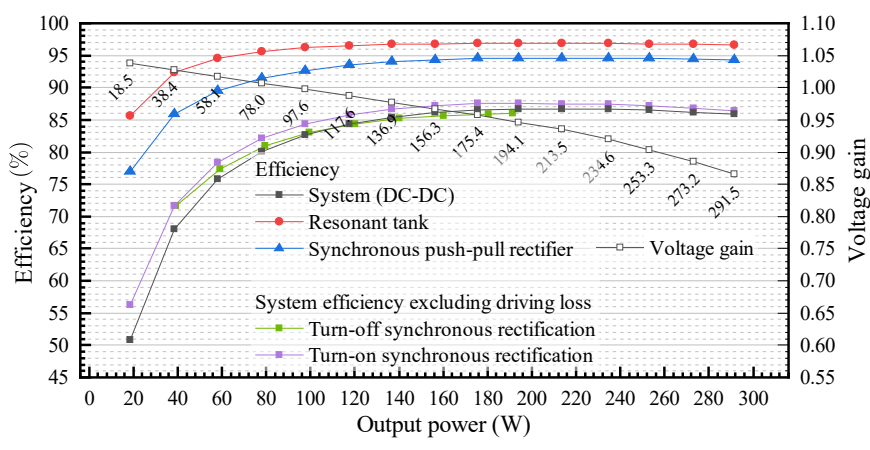

Fig. 16. Measured efficiency and voltage gain $\left(V_{d c o} / V_{d c i}\right)$ versus output power (labeled). The output voltage is fixed at about $48 \mathrm{~V}$ by adjusting the input voltage. There is no external auxiliary DC source applied to the circuit board.

Eventually, the maximum output power is limited by thermal distribution. The measured gain decreases slowly as the output power increases due to the ESRs and nonlinear parasitic capacitance. The decrease accelerates when the output power is above 200 watts. As a solution, feedback control, which is not discussed in this paper, can be adopted to obtain a precise output voltage.

Fig.16 also makes a comparison of the system efficiency with turn-on and turn-off synchronous rectification. Turning off the synchronous driving pulses will result in a different diode-based rectification mode. The two rectification modes are different in terms of duty cycle, input impedance, voltage gain, drain-source voltage, etc. For the diode-based rectification, the driving circuit is not needed. When the output power is below 100 watts, the system efficiency with diode-based rectification is slightly higher. However, when the driving loss (about 2 watts) is excluded from the efficiency calculation, the system efficiency with synchronous rectification is always higher over the entire load range. Since the max surface temperature of the switches will exceed $100{ }^{\circ} \mathrm{C}$, the diodebased rectification is not able to output power above 200 watts. Therefore, the synchronous rectification can output much higher power than the diode-based rectification.

\section{CONClusions}

This paper presents the analytical modeling and optimization of the load-independent synchronous class $\mathrm{E}$ rectifiers. An $L C C-S$ compensated MHz-WPT topology, which comprises the push-pull class $\mathrm{E}$ inverter and rectifier with loadindependent operation, is proposed to achieve the fully softswitching and a nearly-constant voltage gain over the entire load range. The improved design of the compensation network is also investigated to provide a design methodology of the proposed topology. The following conclusions can be made.

1) The ideal load-independent synchronous class $E$ rectifiers realize the zero-phase-angle input impedance and the soft-switching over the entire load range with the constant voltage gain. The measured switching behavior matches well with the theoretical analysis.

2) The proposed optimal parameters of the synchronous class $\mathrm{E}$ rectifier realize both of ZVS turn-on and ZCS 
turn-off at the rated output power. The measured efficiency reaches its maximum value (94.6\%) near the designed rated condition. It provides a preferable design method of the synchronous class E rectifiers.

3) The auxiliary coil can indirectly detect the optimal switching phase of the synchronous switches. Benefited from decoupling the auxiliary coil and receiving coil, the phase detection prevents mutual interference between the switches of the synchronous push-pull class E rectifier. It shows high stability in the $\mathrm{MHz}$ region without adding distinguishable loss.

4) The proposed improved design method of the $L C C-S$ compensation network is conducive to improve the system efficiency. The measured system efficiency reaches its peak value $(86.7 \%, 214$ watts) near the designed rated output power. It matches well with the theoretical optimal loaded quality.

\section{REFERENCES}

[1] Z. Zhang, H. Pang, A. Georgiadis, and C. Cecati, "Wireless power transfer-An overview," IEEE Trans. Ind. Electron., vol. 66, no. 2, pp. 1044-1058, Feb. 2019.

[2] C. Liu, C. Jiang, J. Song, and K. T. Chau, "An effective sandwiched wireless power transfer system for charging implantable cardiac pacemaker," IEEE Trans. Ind. Electron., vol. 66, no. 5, pp. 4108-4117, May 2019.

[3] T. Kan, Y. Zhang, Z. Yan, P. P. Mercier, and C. C. Mi, "A rotationresilient wireless charging system for lightweight autonomous underwater vehicles," IEEE Trans. Veh. Technol., vol. 67, no. 8, pp. 6935-6942, Aug. 2018.

[4] Y. Dou, D. Zhao, Z. Ouyang, and M. A. E. Andersen, "Investigation and design of wireless power transfer system for autonomous underwater vehicle," in Proc. IEEE Appl. Power Electron. Conf., Mar. 2019, pp. 3144-3150.

[5] X. Qu, H. Chu, Z. Huang, S. Wong, C. K. Tse, C. C. Mi, and X. Chen, "Wide design range of constant output current using double-sided LC compensation circuits for inductive-power-transfer applications," IEEE Trans. Power Electron., vol. 34, no. 3, pp. 2364-2374, Mar. 2019.

[6] M. Liu, M. Fu, and C. Ma, "Parameter design for a 6.78-MHz wireless power transfer system based on analytical derivation of class E currentdriven rectifier," IEEE Trans. Power Electron., vol. 31, no. 6, pp. 42804291, Jun. 2016.

[7] M. Liu, M. Fu, and C. Ma, "Low-harmonic-contents and high-efficiency class E full-wave current-driven rectifier for megahertz wireless power transfer systems," IEEE Trans. Power Electron., vol. 32, no. 2, pp. 1198 1209, Feb. 2017.

[8] S. Aldhaher, D. C. Yates, and P. D. Mitcheson, "Load-independent class E/EF inverters and rectifiers for MHz-switching applications," IEEE Trans. Power Electron., vol. 33, no. 10, pp. 8270-8287, Oct. 2018.

[9] K. Li, S. C. Tan, and R. S. Y. Hui, "Single-stage regulated resonant WPT receiver with low input harmonic distortion," IEEE Trans. Power Electron., vol. 35, no. 7, pp. 6820-6829, Jul. 2020.

[10] K. Li, S.-C. Tan, and R. S. Y. Hui, "Single-switch-regulated resonant WPT receiver," IEEE Trans. Power Electron., vol. 34, no. 11, pp. $10386-$ 10391, Nov. 2019.

[11] H. Li, X. Zhang, M. Dai, H. Yang, Y. Wang, and D. Xu, "Design of a wireless power transfer system based on dual-class E self-resonant synchronous rectifier," in Proc. 22nd Int. Conf. on Elect. Machines Syst., Aug. 2019, pp. 1-6.

[12] S. Aldhaher, D. C. Yates, and P. D. Mitcheson, "13.56 MHz $50 \mathrm{~W}$ load-independent synchronous class E rectifier using GaN devices for space-constrained applications," in Proc. IEEE Wireless Power Transfer Conf., Jun. 2018, pp. 1-4.

[13] J. Song, M. Liu, N. Kang, and C. Ma, "A universal optimal drain-sourcevoltage tracking scheme for synchronous resonant rectifiers in megahertz wireless power transfer applications," IEEE Trans. Power Electron., p. 1, 2020.

[14] M. Fu, Z. Tang, and C. Ma, "Analysis and optimized design of compensation capacitors for a megahertz WPT system using full-bridge rectifier," IEEE Trans. Ind. Informat., vol. 15, no. 1, pp. 95-104, Jan. 2019.
[15] Y. Li, X. Ruan, L. Zhang, J. Dai, and Q. Jin, "Optimized parameters design and adaptive duty-cycle adjustment for class E dc-dc converter with on-off control," IEEE Trans. Power Electron., vol. 34, no. 8, pp. 7728-7744, Aug. 2019.

[16] T. Nagashima, X. Wei, E. Bou, E. Alarcón, and H. Sekiya, "Analytical design for resonant inductive coupling wireless power transfer system with class-E inverter and class-DE rectifier," in Proc. IEEE Int. Symp. Circuits Syst., May. 2015, pp. 686-689.

[17] S. Aldhaher, D. C. Yates, and P. D. Mitcheson, "A 25 W $27.12 \mathrm{MHz}$ wireless power transfer system," in Proc. IET Int. Conf. Power Electron. Mach. Drives, Apr. 2016, pp. 1-4.

[18] S. Abbasian and T. Johnson, "High efficiency and high power GaN HEMT inverse class-F synchronous rectifier for wireless power applications," in Proc. European Microwave Conf., Sep. 2015, pp. 299-302.

[19] W. A. Nitz, W. C. Bowman, F. T. Dickens, F. M. Magalhaes, W. Strauss, W. B. Suiter, and N. G. Ziesse, "A new family of resonant rectifier circuits for high frequency dc-dc converter applications," in Proc. IEEE Appl. Power Electron. Conf., Feb. 1988, pp. 12-22.

[20] M. K. Kazimierczuk, "Class E low $\mathrm{dv}_{D} / \mathrm{dt}$ rectifier," IEE Proc. B Electric Power Applicat., vol. 136, no. 6, pp. 257-262, Nov. 1989.

[21] A. Reatti, M. K. Kazimierczuk, and R. Redl, "Class E full-wave low dv/dt rectifier," IEEE Trans. Circuits Syst. I. Fundam. Theory Appl. (1993-2003), vol. 40, no. 2, pp. 73-85, Feb. 1993.

[22] M. K. Kazimierczuk and J. Jozwik, "Class E zero-current-switching rectifier with a series inductor," in Proc. 32nd Midwest Symp. Circuits Syst., Aug. 1989, pp. vol.2, pp. 788-791

[23] J. A. Garcia and Z. Popovic, "Class-E rectifiers and power converters: The operation of the class-E topology as a power amplifier and a rectifier with very high conversion efficiencies," IEEE Microw. Mag., vol. 19, no. 5, pp. 67-78, July-Aug. 2018.

[24] K. Jin, L. Gu, and J. Wang, "A 10-MHz resonant converter with a synchronous rectifier for low-voltage applications," IEEE Trans. Power Electron., vol. 34, no. 4, pp. 3339-3347, Apr. 2019.

[25] Z. Zhang, X. Zou, Z. Dong, Y. Zhou, and X. Ren, "A 10-MHz eGaN isolated class- $\Phi_{2}$ DCX," IEEE Trans. Power Electron., vol. 32, no. 3, pp. 2029-2040, Mar. 2017.

[26] J. A. Santiago-Gonzalez, K. M. Elbaggari, K. K. Afridi, and D. J. Perreault, "Design of class E resonant rectifiers and diode evaluation for VHF power conversion," IEEE Trans. Power Electron., vol. 30, no. 9, pp. 4960-4972, Sep. 2015.

[27] I. Ramos, M. N. Ruiz Lavín, J. A. García, D. Maksimović, and Z. Popović, "GaN microwave dc-dc converters," IEEE Trans. Microw. Theory Tech., vol. 63, no. 12, pp. 4473-4482, Dec. 2015.

[28] X. Huang, Y. Kong, Z. Ouyang, W. Chen, and S. Lin, "Analysis and comparison of push-pull class-E inverters with magnetic integration for megahertz wireless power transfer," IEEE Trans. Power Electron., vol. 35 , no. 1 , pp. 565-577, Jan. 2020.

[29] R. E. Zulinski and K. J. Grady, "Load-independent class E power inverters. Part I. Theoretical development," IEEE Trans. Circuits Syst.* (1974-1992), vol. 37, no. 8, pp. 1010-1018, Aug. 1990.

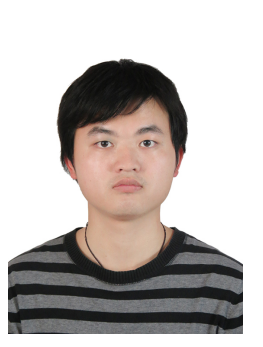

Xiaosheng Huang (M'20) received his B.E. and Ph.D. degree in 2009 and 2015 from Fuzhou University, Fuzhou, China respectively. He is currently working as an associate professor in the School of Electronic, Electrical Engineering and Physics, Fujian University of Technology, Fuzhou, China. He is also with the Fujian Provincial University Engineering Research Center for Industrial Automation, Fujian University of Technology. He is a member of the Magnetic Component Specialty Committee of the China Power Supply Society. His current research interests include power conversion, high-frequency magnetics, wireless power transfer, and electromagnetic field analysis and applications.

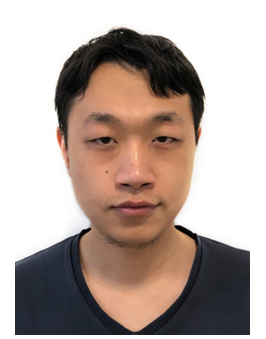

Yi Dou (S'17) received the B.S. degree in electrical engineering from $\mathrm{Xi}^{\prime}$ an Jiaotong University, China and M.Sc. degree in power electronics from Technical University of Denmark, Denmark in 2016 and 2018, respectively. He is currently working toward the Ph.D. degree at Technical University of Denmark. His research focuses on design of high-frequency/ very-high-frequency DC-DC converters, modelling for magnetic components, and modelling and optimization of MHz-range wireless power transfer systems. 


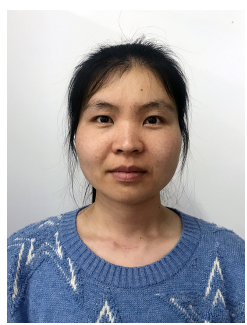

Shuyi Lin received the B.E. and Ph.D. degree in 2008 and 2014 from Fuzhou University, Fuzhou, China respectively. She is currently working as an associate professor in the School of Electronic, Electrical Engineering and Physics, Fujian University of Technology, Fuzhou, China. She is also with the Fujian Provincial University Engineering Research Center for Industrial Automation, Fujian University of Technology. Her current research interests include power conversion, high-frequency magnetics, wireless power transfer.

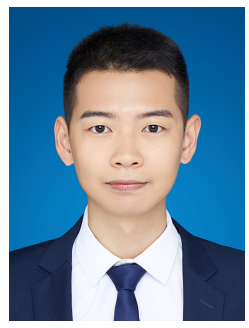

Yuan Tian received the B.E. degree in 2018 from Xiangnan University, Hunan, China. He is currently working toward the M.E. degree in the School of Electronic, Electrical Engineering and Physics, Fujian University of Technology, Fuzhou, China. His research interests include wireless power transfer and optimization of high-frequency magnetics.

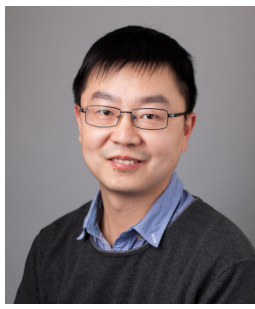

Ziwei Ouyang (S'07, M'11, SM'17) received his $\mathrm{PhD}$ degree from Technical University of Denmark (DTU) in 2011. From 2011 to 2013, he was a postdoc researcher at DTU. From 2013 to 2016, he was appointed as an assistant professor at the same department. Since from April 2016, he is an associate professor at DTU. His research areas focus on high-frequency planar magnetics modeling and integration, high-density high efficiency power converters, PV battery energy storage system, and wireless charging etc. He is IEEE senior member. He has over 70 high impact IEEE journal and conference publications, co-author on a book chapter on Magnetics for the "Handbook of Power Electronics" and currently he is the holder of 8 international patents. He was a recipient of Young Engineer Award at PCIM Asia 2014, and received Best Ph.D. Dissertation of the Year Award 2012 in Technical University of Denmark. He received several Best Paper Awards in IEEE sponsored international conferences. He has been invited to give lectures in many universities, enterprises and educational seminars and workshops around the world including USA, Europe and China. He has served as session chair in some IEEE sponsored conferences and associated editor for IEEE Journal of Emerging and Selected Topics in Power Electronics.

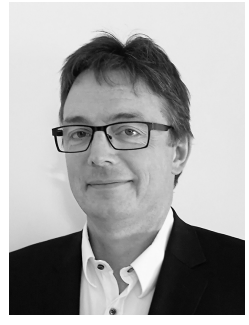

Michael A. E. Andersen (M'88) received the M.Sc.E.E. and Ph.D. degrees in power electronics from the Technical University of Denmark, Kongens Lyngby, Denmark, in 1987 and 1990, respectively. He is currently a Professor of power electronics at the Technical University of Denmark, where since 2009, he has been the Deputy Head of the Department of Electrical Engineering. He is the author or coauthor of more than 300 publications. His research interests include switch-mode power supplies, piezoelectric transformers, power factor correction, and switch-mode audio power amplifiers. 